of Australopithecus, but reduced in size and degenerate. The molars of the gorilla and chimpanzee are probably evolved from a pre-Australopithecus type.

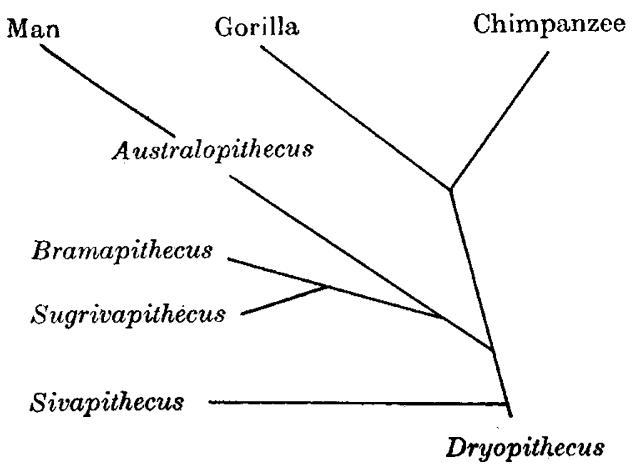

All the available evidence would seem to indicate that the higher anthropoids and man may be related as shown in the accompanying diagram.

Transvaal Museum,

R. Broom.

Pretoria,

South Africa.

Sept. 21.

\section{Detection of $\alpha$-Particles in the Disintegration of Thorium}

THE nuclear process $(n \alpha)$, that is, the expulsion of an $\alpha$-particle by a neutron, has hitherto been observed only for light nuclei ; it probably takes place also in the case of heavy, unstable nuclei. Hahn, Meitner ${ }^{1}$ and Curie, v. Halban and Preiswerk ${ }^{2}$ assumed the reaction ${ }_{90}^{232} \mathrm{Th}(n \alpha){ }_{88}^{229} \mathrm{Ra}$, and the existence of the radium isotope was established by the latter three by chemical identification.

We have made an attempt to detect directly the presence of these $\alpha$-particles, which we expected to be of high energy. We used an ionization chamber in conjunction with a linear amplifier. When thorium was bombarded by neutrons from a radium-beryllium source, $\alpha$-particles were detected, which must be ascribed to the reaction ${ }_{90}^{232} \mathrm{Th}(n \alpha){ }_{88}^{229} \mathrm{Ra}$. Preliminary measurements with absorbing screens have shown that these $\alpha$-particles possess an energy greater than 9 million electron volts.

A full account of these experiments will appear in due course in Helv. phys. Acta.

Physikalisches Institut,
A. Braun.
P. Preiswerk.
P. SCHERRER.

Eidgenössische Technische Hochschule, Zürich. Aug. 31.

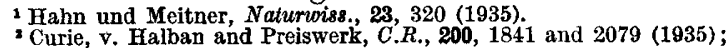
J. Phys., 6, 361 (1935).

\section{Production of Showers by Heavy Particles}

Carlson and Oppenheimer ${ }^{1}$ and Bhabha and Heitler ${ }^{2}$ have proposed a very ingenious explanation of showers by a multiplicative production of photons, electrons and positrons. The greater part of the showers observed can in this way be regarded as originated by electrons, positrons or photons penetrating from the atmosphere. Such showers are absorbed if the layer of lead is sufficiently thick. It is known, however, that at sea-level and especially under earth, the frequency of showers with increasing thickness of lead layer does not diminish but tends to a limit. We wish to point out that this phenomenon does not require a new concept of showers, but can be explained by irradiation with heavy particles, which easily penetrate through such layers of lead.

A more rigorous treatment of showers ${ }^{3}$ shows that a shower has a certain range given by the formula

$$
L=0.25 \frac{\hbar c}{e^{2}}\left(\frac{m c^{2}}{e^{2}}\right)^{2} \frac{1}{\bar{Z} N} \log \frac{E}{\varepsilon},
$$

where $\hbar, c, e$ have the usual meaning; $m$ is the electronic mass ; $N$ is the number of electrons per cm. $^{3} ; \bar{Z}=\frac{\Sigma N_{z} Z(Z+1)}{\Sigma N_{z} Z}\left(N_{z}\right.$ is number of atoms with charge $Z e$ ); $E$ is the energy of the incident particle and $\varepsilon$ a critical energy of the order of magnitude $750 / \bar{Z}$ million volt.

If we combine this formula with the well-known formula for radiation probability for a heavy particle, we get for the probability that a shower produced by a photon emitted by a heavy particle gets out of the layer of sufficient thickness:

$$
W=0.8\left(\frac{m}{M}\right)^{2}\left(\log \frac{E}{\varepsilon}\right)^{2} \log \frac{E}{\left(M c^{2}\right)^{3 / 4} \varepsilon^{1 / 4}} ;
$$

where $M$ is the mass of the heavy particle and $E$ its energy, which is assumed $\gg M c^{2}$. If we take for $M$ the value of some tens of electronic mass proposed by Anderson and Neddermeyer ${ }^{4}$, we get a fair agreement with the observed order of magnitude.

Details of the calculations will be published elsewhere.

\section{Physical Institute,}

L. LANDAU.

Academy of Sciences of the U.S.S.R.,

G. RUMER.

Moscow.
Sept. 1.

${ }^{1}$ Phys. Rev., 51, 220 (1937).

${ }^{2}$ Proc. Roy. Soc., A, 159, 432 (1937).

${ }^{3}$ Phys. Z. Sovjetunion, in the Press.

'Phys. Rev., 51, 887 (1937).

\title{
The Zodiacal Light at a Total Solar Eclipse
}

As interesting observation made by Mr. M. Honda in Hokkaido at the total solar eclipse of June 19, 1936, has just been reported and is well worth mention.

Seated in a lightproof bag, which covered his head also until totality had commenced, Mr. Honda observed the sky round the sun, with a large black disk blotting out an area of radius about three solar diameters with the sun at the centre. He saw and plotted the boundaries of the band of zodiacal light from close to the sun, where it had a width of $44^{\circ}$, along the ecliptic to a point about $40^{\circ}$ away where it was cut off by clouds. To see the zodiacal light, on what is effectively a moonlit sky, is difficult, but the band must be very much brighter near the sun. Mr. Honda's observation is well worth repeating by other experienced zodiacal light observers at the next eclipse, and if possible some quantitative measure of the intensity of the band should be obtained along with measurements of coronal intensity.

Solar: Physics Observatory,

F. J. M. St'tratton. Cambridge. 